\title{
Neurology podcast utilization during the COVID-19 pandemic
}

\author{
James E. Siegler ${ }^{1,2}\left(\right.$ D $\cdot$ Patrick E. Boreskie ${ }^{2} \cdot$ Roy Strowd $^{3} \cdot$ Robert Rook $^{4} \cdot$ Adeline Goss $^{5} \cdot$ Fawaz Al-Mufti $^{6}$. \\ Bonnie Rossow ${ }^{7} \cdot$ Alexandra Miller $^{8} \cdot$ Amanda Chamberlain $^{4} \cdot$ Zachary London $^{9} \cdot$ Jennifer Hurley $^{10}$. \\ Romergryko Geocadin ${ }^{11} \cdot$ Megan Richie ${ }^{5} \cdot$ Richard Isaacson $^{12} \cdot$ Igor Rybinnik $^{13} \cdot$ Teresa M. Chan $^{14}$
}

Received: 10 March 2021 / Accepted: 31 July 2021 / Published online: 12 August 2021

(c) Fondazione Società Italiana di Neurologia 2021

\begin{abstract}
Background As medical education shifted to a virtual environment during the early coronavirus disease 2019 (COVID-19) pandemic, we evaluated how neurology podcasting may have been utilized during this period, and which features of podcasts have been more highly sought by a medical audience.

Methods We conducted a retrospective analysis of neurology-themed blogs and/or podcasts between April 2019 and May 2020. Programs were eligible if they reported mean monthly downloads $>2000$, were affiliated with an academic society, or offered continuing medical education credit. Thirty-day download counts were compared between study months, with adjustment for multiple testing. Exploratory analyses were performed to determine which podcast features were associated with higher downloads.

Results Of the 12 neurology podcasts surveyed, 8 completed the survey and 5 met inclusion criteria. The median monthly download count was 2865 (IQR 869-7497), with significant variability between programs $(p<0.001)$. While there was a $358 \%$ increase in downloads during April 2020 when compared to the previous month, this was not significant (median 8124 [IQR 2913-14,177] vs. 2268 [IQR 540-6116], $\left.p_{\text {adj }}=0.80\right)$. The non-significant increase in overall downloads during April 2020 corresponded to an increase in unique episodes during that month $(r=0.48, p=0.003)$. There was no difference in 30-day downloads among episodes including COVID-19 content versus not (median 1979 [IQR 791-2873] vs. 1171 [IQR 405-2665], $p=0.28)$.

Conclusions In this unique, exploratory study of academic neurology-themed podcasts, there was no significant increase in episode downloads during the early COVID-19 pandemic. A more comprehensive analysis of general and subspecialty medical podcasts is underway.
\end{abstract}

Keywords Podcasting $\cdot$ COVID-19 $\cdot$ Medical education

James E. Siegler

siegler-james@cooperhealth.edu

1 Cooper Neurological Institute, Cooper University Hospital, 3 Cooper Plaza Suite 320, Camden, NJ 08103, USA

2 Department of Emergency Medicine, Max Rady College of Medicine, Rady Faculty of Health Sciences, University of Manitoba, Winnipeg, Manitoba, Canada

3 Department of Neurology, Wake Forest School of Medicine, Winston Salem, NC 27157, USA

4 American Academy of Neurology, Minneapolis, MN 55415, USA

5 Department of Neurology, University of California San Francisco, San Francisco, CA 94143, USA

6 Westchester Medical Center at New York Medical College, Valhalla, NY 10595, USA
7 Neurocritical Care Society, Chicago, IL, USA

8 Memorial Sloan Kettering, New York, NY 10065, USA

9 Department of Neurology, University of Michigan, Ann Arbor, MI 48109, USA

10 American Neurological Association, Mount Laurel, NJ, USA

11 Department of Neurology, Johns Hopkins Medical Institute, Baltimore, MD 21287, USA

12 Department of Neurology, New York Presbyterian/Weill Cornell Medicine, New York, NY 10021, USA

13 Department of Neurology, Rutgers Robert Wood Johnson Medical School, New Brunswick, NJ 08901, USA

14 Division of Emergency Medicine and Division of Education \& Innovation, Department of Medicine, McMaster University, Hamilton, Ontario, Canada 


\section{Introduction}

The continuing provision for formal medical education is one of the many challenges faced by educators and administrators during the coronavirus disease 2019 (COVID19) pandemic [1]. Although this particular challenge was vocalized by educators and trainees in the spring of 2020 [2-5], the pandemic remains a major barrier to the successful training of medical providers [6]. Early recommendations by the Liaison Committee on Medical Education to suspend medical student involvement from patient-related activities compounded by ongoing virtual didactics were only a few contributors to the widespread implementation of self-directed graduate medical education [3]. Reductions in hospital presentations and outpatient neurology visits $[7,8]$ have also scaled back the clinical experience among trainees [9]. Furthermore, the cancellation (or limitation) of academic conferences has curtailed many opportunities for education, networking, and career development among medical providers. The advantages and disadvantages of a transition to a highly virtual learning environment (e.g., using Online Educational Resources, OERs) have been summarized previously [10,11], but not all programs have been able to convert their curricula quickly and effectively in response to the pandemic. Established OERs in the form of online publications and video and audio podcasts have provided healthcare professionals [12] and educators [13] with the opportunity to incorporate existing resources into a developing digital syllabus. Anecdotal reports indicate a heightened use of widely available content via social media, virtual grand rounds, webinars, and other resources-even before the advent of the COVID-19 pandemic [14].

Originally a grassroots movement, the creation of medical OERs has blossomed over the last two decades, with numerous examples of multi-author websites, resources sponsored or supported by medical associations and journals, and highly networked learning environments populated by all levels of medical education learners [15]. In neurology, podcasting has played a major role in building OER popularity over the past 10 years $[16,17]$. The first academically designed, neurology-themed podcast was launched in 2006 by The Lancet Neurology. This inaugural program was followed in rapid succession by podcasts from the American Academy of Neurology (2007), the Journal of the American Medical Association Neurology (2011), the Journal of Neurology, Neurosurgery, and Psychiatry (2013), the Neurocritical Care Society (2017), and the American Neurological Association (2019), among other academic neurology societies and independent groups.

The reach and utilization of neurology-themed podcasts, however, have never been formally investigated. As many educators have turned to (and trainees have grown reliant upon) digital resources during the COVID-19 pandemic, the ADVANCE (Advocates for Digital, Visual, Audio, and Networked Clinical Education) investigators sought to explore how podcasts have filled this gap in medical education during the early months of COVID- 19 . As a secondary analysis of these data, we explored which programs and episode features garnered the greatest attention in the form of unique download counts. Awareness of popularity trends and program features may help guide future educators when producing their own digital educational syllabi, or selecting which existing programs may be most useful to incorporate into an existing curriculum.

\section{Methods}

\section{Definitions}

For the purposes of this study, a "participant" is defined as any producer, author, or editor of digital content that was generated for the primary purpose of medical education. "Programs" refer to the podcast, with "episode" referring to each unique podcast audio publication. Access to published content was summated in the form of "downloads" (the number of user requests to download, livestream, or otherwise play a particular podcast episode).

\section{Study design}

Data will be made available to any qualified researcher upon reasonable request. We conducted a retrospective, observational study of neurology-specific OER developers as a subset of the more broad ADVANCE dataset. The main results of ADVANCE will be published separately. Participants who produced either (1) audio and/or video podcasts or (2) blogs for the purposes of medical education (April 01, 2019-May 31, 2020) were recruited based on affiliation with the ADVANCE principal investigators (JES, PB, and TC) for related studies on blogs and podcasts [16, 17], or were invited to participate based on affiliation with an academic society and/or peer-reviewed medical journal. Additionally, participation in the ADVANCE study was offered to any qualified producer, author, or editor by way of social media posting and word-of-mouth. Participants were not financially compensated for their contribution to the ADVANCE study. See Fig. 1 for inclusion flowchart.

Neurology programs were included if they (1) reported a mean view/download count of greater than 2000 per month for their blog or podcast during 2019 (set as a minimum standard for notable impact for publications, as described previously [18]), (2) they were affiliated with an academic society or peer-reviewed medical journal, or (3) they offer 


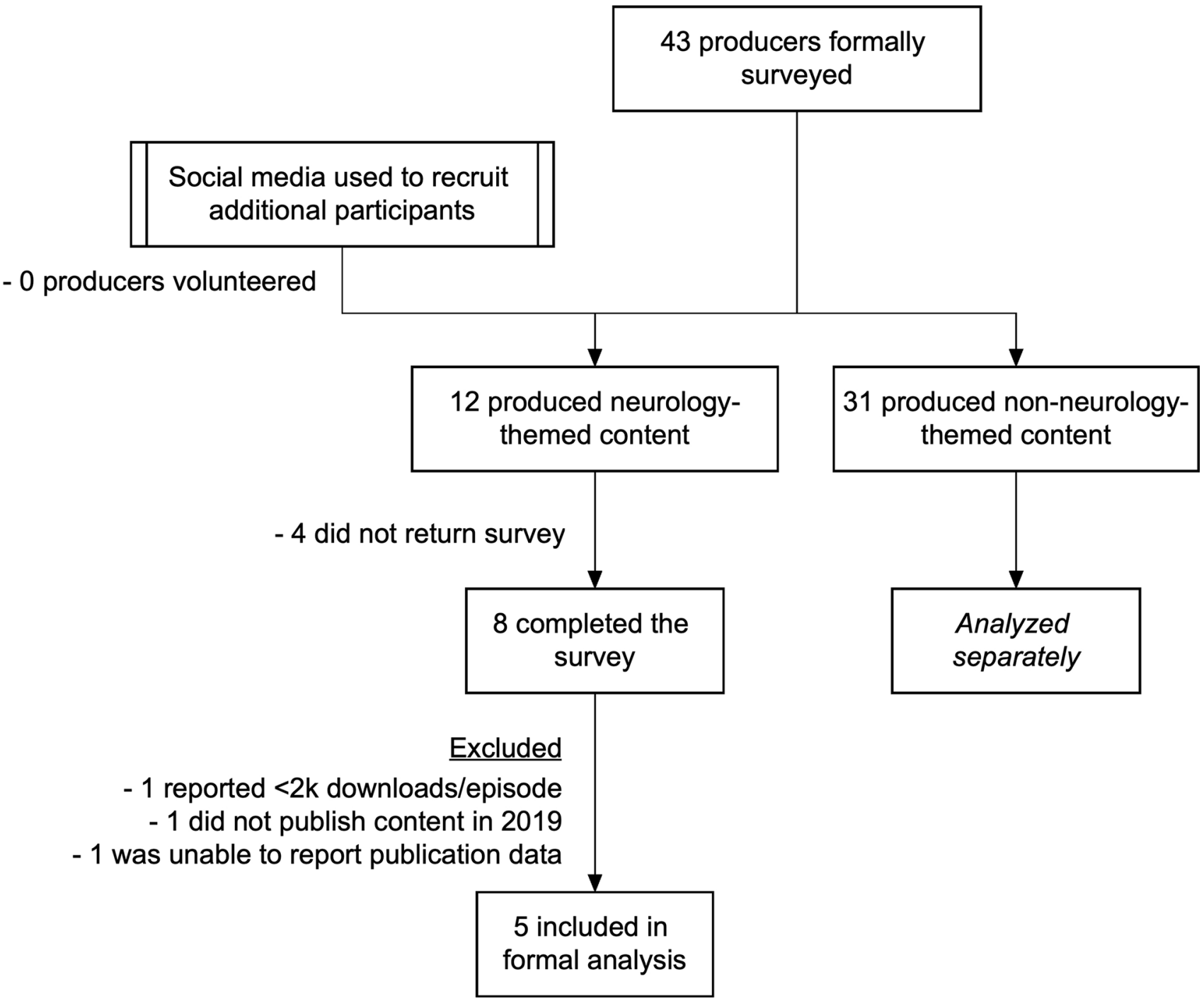

Fig. 1 Inclusion flowchart

continuing medical education (CME) credit to their users. These criteria were set as a surrogate for impact, with traffic counts (unique downloads) in line with previously published setpoints for academic advancement and impact [18]. Programs were excluded if the target audience did not include practicing medical professionals, students from the medical field, or medical graduate/postgraduate trainees. Programs were also excluded if they did not publish content prior to January 1, 2020, or were unable to provide data for the study period.

A brief survey instrument was circulated to all participants in order to determine eligibility for inclusion in ADVANCE. If participants confirmed they met inclusion criteria and lacked exclusion scenarios, they were invited to submit detailed data regarding consecutive episodes including the primary medical specialty of their program (e.g., internal medicine, neurology), target audience (practicing professionals, physician trainees, students, other), social media presence, affiliation with an academic society or peer-reviewed medical journal, unique podcast episode downloads, and provision of CME credit for use of their program. Participants were also asked to provide the title and topic of individual publications and whether or not the episode included COVID-19 content. Due to the popularity and versatility of the platform (e.g., typewritten and graphic content, embedding of links), Twitter accounts and followers were used to characterize social media affiliation and reach. In order to protect anonymity of each program, the number of Twitter followers for each program are reported categorically $(<1 \mathrm{k}, 1-5 \mathrm{k}, 5-10 \mathrm{k}$, 10-25 k, 25-50 k, > 50 k). All data were self-reported by participants, with Twitter data confirmed by the senior author (JES) as of December 20, 2020. 


\section{Statistical analysis}

The primary outcome was the number of unique podcast episode downloads. Secondary outcomes included the number of downloads per unique program, unique downloads per episode, and the monthly downloads per program. Because users may have utilized several platforms in order to access content (e.g., personal computer, smart phone, tablet, or smart home device), it was not feasible to tabulate episode downloads for a given user. Therefore, episode downloads were aggregated for each unique publication across all users. One program published early content (in 2019) using a subscription-based OER platform, and later transitioned much of its 2020 content to a free open-access medical education (FOAM) platform. Cumulative data from the subscriptionbased and FOAM-based platforms were included in the formal analyses.

Descriptive statistics were used for groupwise comparisons. Categorical data were compared using chi square test, or Fisher's exact test when contingency table cell counts were 5 or less. Normality of continuous data was assessed histographically and confirmed using the Shapiro-Wilk test. Downloads were summated over the 24 -h period after release and for the 30-day period following their release, as these are the most commonly reported indices of accessed program content on commercially available Rich Site Summary feeds.

In order to optimize sensitivity for capturing a difference in downloads during the COVID-19 pandemic, we performed 2 comparisons. First, downloads were compared between all months using the Kruskal-Wallis equality of populations rank test, with Dunn's pairwise correction using the Holm-Sidák method for multiple testing as previously described [19]. Second, downloads were compared between the immediate pre-COVID-19 months (January-March 2020 vs. April-May 2020) using the Wilcoxon signed-rank test, with pre-specified subgroup analyses of 30-day downloads stratified according to provision of CME credit, affiliation with academic society or peer-reviewed journal, episode format (interview with author, topical review, or other), and target audience (trainees vs. all other). The Pearson correlation coefficient was used to estimate the relationship between overall downloads, total episodes, and monthly episodes. A word cloud was generated using freely available online software in which the more frequently used words in episode titles corresponded with larger words in the titles (WordArt. com).

All analyses were conducted using STATA 15.0 (College Station, TX) on the two-sided level. $p$ values are provided for convention with a significance level set at 0.05 .

This study did not meet criteria for human subjects research; therefore, approval by the Institutional Review Board of the data coordinating center (Cooper University Hospital) was not required.

\section{Results}

Of the 43 surveyed producers, 12 were producers and/or editors of neurology-themed blogs and/or podcasts, 8 of whom completed the survey for this pre-specified subgroup analysis (66.7\% response rate). Five programs met formal inclusion criteria (Fig. 1), and all were podcasts. Although social media posting and word-of-mouth were utilized to passively recruit additional participants, there were no other respondents recruited in this manner. Four programs had formal affiliations with an academic society and a peerreviewed neurology journal (median 2019 impact factor of 8.55 ) and 1 program was independently published without formal affiliation with an academic society or peer-reviewed journal (Table 1).

\section{Monthly downloads}

The median monthly download count was 2865 (IQR 869-7497) among podcasts, with significant differences in downloads between programs $(p<0.001 ;$ Fig. 2$)$. While there was a $358 \%$ increase in total downloads during April

Table 1 Program descriptions

\begin{tabular}{|c|c|c|c|c|c|c|}
\hline $\begin{array}{l}\text { Anonymized } \\
\text { program }\end{array}$ & $\begin{array}{l}\text { Affiliation } \\
\text { with academic } \\
\text { society }\end{array}$ & $\begin{array}{l}\text { Affiliation with } \\
\text { peer-reviewed } \\
\text { journal }\end{array}$ & Twitter profile (followers) & CME offered & $\begin{array}{l}\text { Unique episodes } \\
\text { (Jan - May 2020) }\end{array}$ & $\begin{array}{l}\text { Median downloads/ month } \\
\text { (IQR) }\end{array}$ \\
\hline 1 & No & No & Yes $(1-5 \mathrm{k})$ & No & 13 & $21,666(19,758-22,547)$ \\
\hline 2 & Yes & Yes & Yes $(25-50 \mathrm{k})$ & No & 5 & $6235(4165-7497)$ \\
\hline 3 & Yes & Yes & Yes $(5-10 \mathrm{k})$ & Yes & 7 & $2681(2268-2955)$ \\
\hline 4 & Yes & Yes & Yes $(25-50 \mathrm{k})$ & Yes & 11 & $668(287-1980)$ \\
\hline 5 & Yes & Yes & Yes $(5-10 \mathrm{k})$ & No & 8 & $521(126-869)$ \\
\hline
\end{tabular}

$C M E$ denotes continuing medical education, and $I Q R$ interquartile range

More detailed information regarding journal impact factor, social media presence and following, and other data not shown in order to protect anonymity of each program 

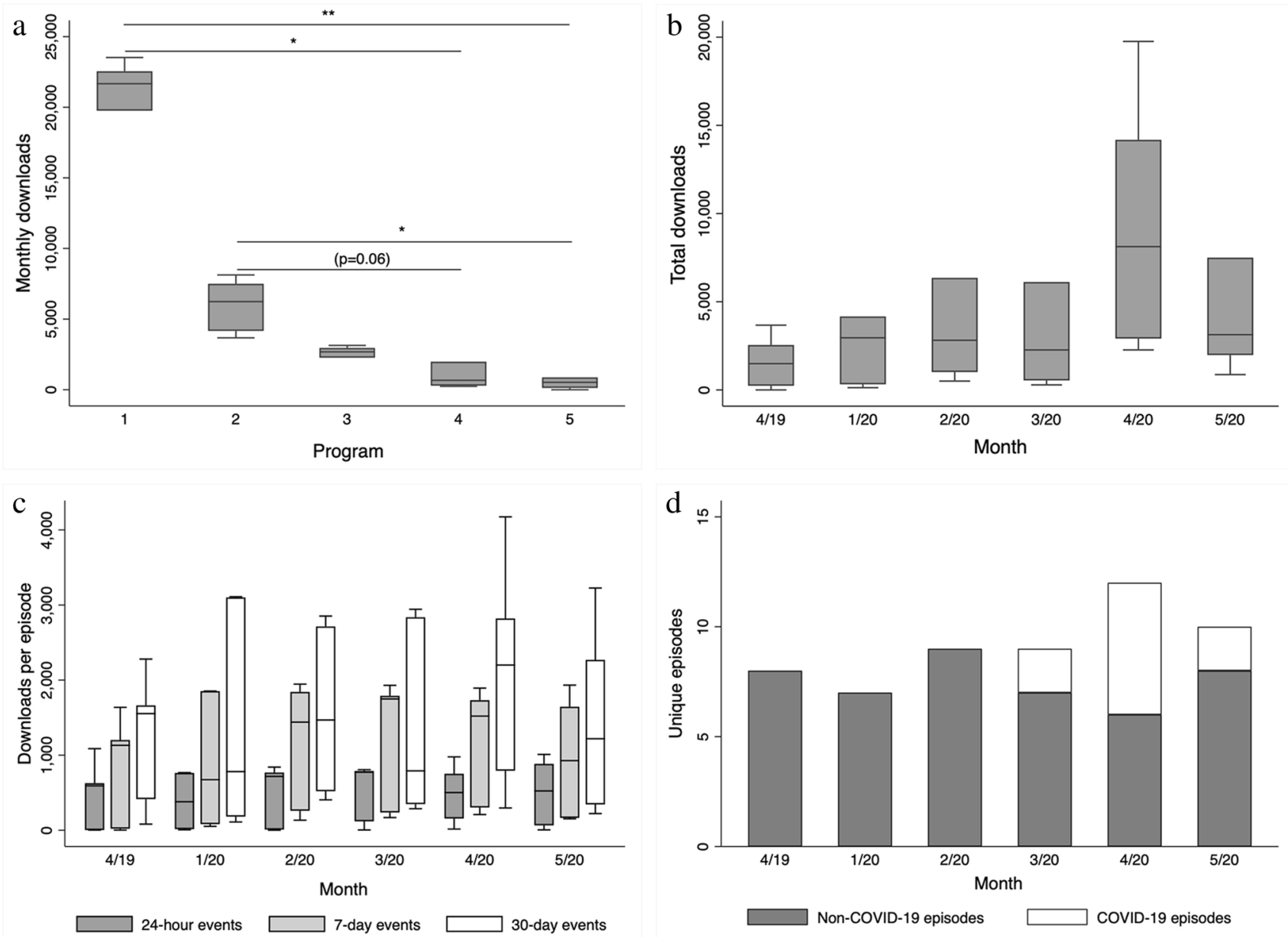

Fig. 2 Download counts by program and month. A Monthly downloads (median with interquartile range) stratified by program. B Program downloads (median with interquartile range) stratified by month. Although there is a visible increase in monthly downloads (episode downloads), there were no significant differences appreciated between month-to-month comparisons. C Downloads within 24-h, 7-day, and 30-day periods following date of episode publication. Episodes are grouped by month according to date of publication

2020 when compared to the previous month (median 8124 [IQR 2913-14,177] vs. 2268 [IQR 540-6116], $p_{\text {adj }}=0.80$; Fig. 2B), this did not achieve statistical significance in unadjusted groupwise comparisons $(p=0.45)$ or adjusted pairwise comparisons ( $p_{\text {adj }}>0.05$ for all pairwise comparisons). When comparing downloads from April 2020 versus April 2019 (median 1490, [IQR 238-2546]), there remained no statistically significant difference despite there being a $545 \%$ increase $\left(p_{\text {adj }}=0.27\right)$. All programs reported an increase in downloads in April 2020 versus the seasonal control month (April 2019) and versus the preceding month (March 2020). In May of 2020, there was a non-statistically significant, but visible normalization of download counts. (e.g., an episode that occurred on March 17, 2020, would be grouped in the March 2020 period). Although there was a small, visible increase in 30-day downloads during April 2020, there were no significant differences in downloads across 24-h, 7-day, or 30-day periods. D Unique episodes by month, stratified by presence or absence of COVID-19 content. ${ }^{*} p \leq 0.01$; ${ }^{*} p \leq 0.001$. All $p$-values shown here are adjusted using the Holm-Sidák method. COVID-19 denotes coronavirus disease 2019

\section{Episode characteristics}

Fifty-two unique episodes were published during the study period. All episodes included content that was directed at practicing professionals $(100 \%)$, while $88 \%$ of episodes included content intended for residents, fellows, and medical students, 58\% intended for patients, and 58\% also intended for non-medical listeners.

There was no significant difference in downloads per unique episode when compared across months in adjusted or unadjusted analyses (unadjusted $p_{24 \mathrm{~h}}=0.91, p_{7 \mathrm{~d}}=0.57$, $p_{30 \mathrm{~d}}=0.63$; all adjusted pairwise $p>0.05$; Fig. 2C). The non-significant increase in overall downloads during April 2020 corresponded to an increase in unique episodes during 
that month ( $r=0.48, p=0.003)$, but bore no relationship to the number of downloads per unique episode.

\section{Subgroup analyses}

Although there was no significant increase in 30-day download counts during the COVID-19 months versus the preceding months, one program (anonymized program no. 4) reported significantly greater downloads per unique episode. This was mediated by the program's transition for some of its content from a subscription-based OER to a FOAMbased (Free Open Access Medicine) OER. By increasing the accessibility of its content to any user, the number of 30-day downloads increased by a mean of 2247 per unique episode that was made freely available (a mean growth of $375 \%$ per episode). The increase in download numbers attributed to this free OER medium (YouTube) showed continued growth over time as more episodes were published. The first episode accumulated 591 additional downloads over 30 days (99\% gain) and the third episode accumulated 5280 additional downloads (605\% gain).

CME credit was made available by 2 programs ( $31 \%$ of episodes), but 30-day downloads were $73 \%$ lower for episodes in which CME was offered (median download count 602 [IQR 323-762] vs. 2210 [IQR 1171-2845], $p<0.001$ ).
There was no difference in 30-day downloads during the months of COVID-19 based on availability of CME credit (Table 2).

Four of the five included programs were affiliated with an academic society or peer-reviewed journal, which reported significantly lower 30-day downloads than the program which was not affiliated with an academic society or journal (median 734 [IQR 297-1881] vs. 2769 [IQR 2551-2888], $p<0.0001)$. There was a trend toward higher download counts during the COVID-19 months versus the preceding months among programs with an academic society affiliation ( $p=0.06$; Table 2$)$, which was driven by anonymized program no. 4 for reasons outlined above.

The most frequently cited format for episodes was "topical review" (76\%), followed by "interview with investigator/author" (11\%) and other/unspecified (13\%). There were non-significantly higher 30-day downloads among episodes designated as interviews (median 2210 [IQR 2157-2269]) when compared to reviews (1489 [IQR 405-2726]) and other episodes (428 [IQR 222-2943]; $p=0.31$ ). There was no difference in 30-day downloads during the months of COVID-19 based on episode format (Table 2).

The majority of episodes included content that targeted medical trainees (88\%), but there was no significant difference in 30-day downloads based on whether episodes
Table 2 Subgroup analysis of 30-day downloads

\begin{tabular}{|c|c|c|c|}
\hline & $\begin{array}{l}\text { January-March } 2020 \\
\text { ( } n=22 \text { episodes })\end{array}$ & $\begin{array}{l}\text { April-May } 2020 \\
(n=25 \text { episodes })\end{array}$ & $p$-value \\
\hline All & $786(734-2769)$ & 1969 (734-2769) & 0.44 \\
\hline \multicolumn{4}{|l|}{ Anonymized program no } \\
\hline 1 & $2845(2715-3022)$ & $2873(2769-2888)$ & 0.77 \\
\hline 2 & $2038(1881-2195)$ & $2225(2157-2269)$ & 0.25 \\
\hline 3 & $762(636-786)$ & $740(734-822)$ & 1.00 \\
\hline 4 & $287(209-405)$ & 852 (1699-4174) & 0.02 \\
\hline 5 & $348(182-428)$ & $297(231-374)$ & 0.88 \\
\hline \multicolumn{4}{|l|}{ CME credit } \\
\hline Not offered & $2551(405-2853)$ & $2467(1781)$ & 0.38 \\
\hline Offered & $529(348-781)$ & $554(264-781)$ & 0.91 \\
\hline \multicolumn{4}{|c|}{ Peer-reviewed journal or academic society affiliation } \\
\hline No & $2845(2715-3022)$ & $2873(2769-2888)$ & 0.77 \\
\hline Yes & $479(287-781)$ & $852(374-2176)$ & 0.06 \\
\hline \multicolumn{4}{|l|}{ Episode format } \\
\hline Topical review & $742(348-2726)$ & $1781(740-2873)$ & 0.18 \\
\hline Interview with author & 2038 (1881-2195) & 2225 (2157-2269) & 0.25 \\
\hline Other & $791(428-2943)$ & $260(222-297)$ & 0.25 \\
\hline \multicolumn{4}{|l|}{ Target audience } \\
\hline $\begin{array}{l}\text { Trainees (residents/fellows, } \\
\text { students) }\end{array}$ & $762(377-2782)$ & $1616(374-2873)$ & 0.42 \\
\hline Non-trainees & 2038 (1881-2195) & $2225(2157-2269)$ & 0.25 \\
\hline
\end{tabular}

CME denotes continuing medical education

Data are shown as median downloads per unique episode with interquartile range 
targeted trainees or not (median 837 [IQR 374-2726] vs. 2210 [IQR 2157-2269], $p=0.18$ ). Similarly, there was no significant difference in 30-day downloads during the months of COVID-19 when episodes were stratified according to target audience being trainees or non-trainees (Table 2).

While the most common, singular term included in episode titles was "COVID" $(n=6 ; 10$ episodes featuring COVID-19 content; Fig. 3), there was no difference in 30-day downloads among episodes including COVID-19 content versus not (median 1979 [IQR 791-2873] vs. 1171 [IQR 405-2665], $p=0.28$ ).

\section{Discussion}

In the wake of the global response to COVID-19, there was a slight, but non-significant increase in neurology podcast utilization as compared to previous months. The increase was also evident, although not statistically significantly different, when compared to the identical month in the preceding year. This increase in podcast utilization appears driven by the increase in the number of published episodes during April 2020 rather than the increase in unique episode downloads. Therefore, we cannot conclude that there was any significant increase in usage of podcast-based medical education during the COVID-19 pandemic. Unique episode download counts were unchanged during the early phase of the COVID-19 pandemic, and although there is no way to confirm this, it is most likely that users continued to access program content with similar frequency during the pandemic as in preceding months.

As a secondary objective to this analysis, we sought to determine which program or episode features were associated with the greatest number of unique downloads. When podcast episodes were organized according to the type of content (e.g., COVID-19-related or not, provision of CME credit, and format of episode), there was no significant difference indicating one episode type was more popular than any other. That said, there were numerically more downloads per podcast episode among episodes structured as "interview with author." This may indicate that listeners prioritize investigator credibility, peer-review of published written material, and evidence-based medical practice. Many of these features have already been established as quality indicators in blogs and podcasts [20]. However, the fact that the program with highest monthly downloads was not affiliated with a peer-reviewed journal or academic society (and that it published content exclusively categorized as "topical review" or "other") suggests that episodes featuring author interviews may not be the only route to a popular program. It is possible that, although the target audiences are likely similar across each program, the individual listeners may differ.

Of note, one podcast began to move some of its content from an entirely subscription-based OER to a FOAM-based OER. By offering content to users for free, the number of 30-day downloads increased by an average of more than 2200 per unique episode. The gains in download numbers

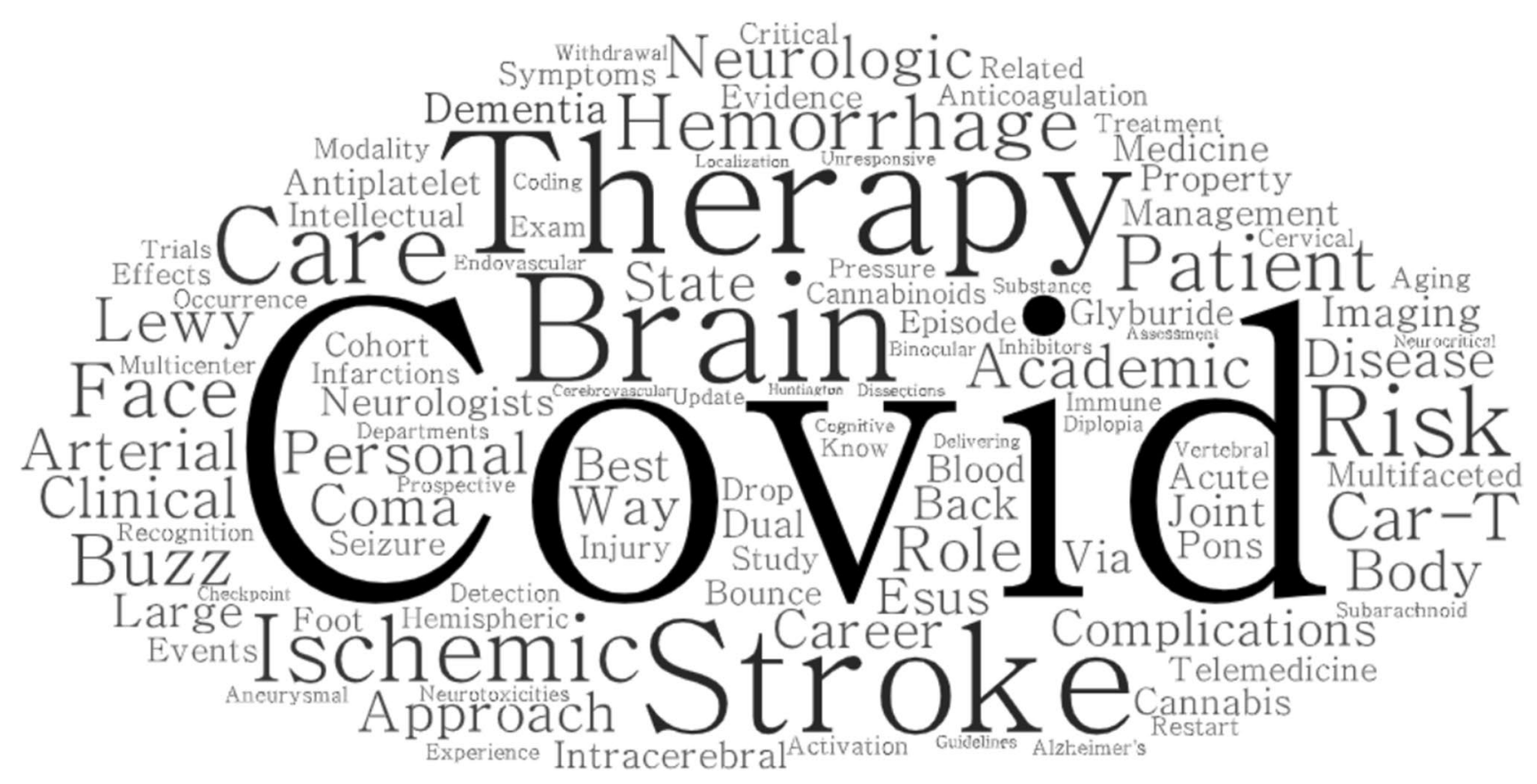

Fig. 3 Word cloud generated from the most frequent text found in episode titles 
showed continued growth as more episodes were published to YouTube, and should be expected to continue climbing as new users subscribe to this YouTube channel. At this time, this program does not plan to move all of its content to a FOAM-based OER medium, but is leveraging this platform to provide "general neurology" content to non-neurology users who might otherwise not have accessed their program's content. Importantly, while content from this program was more accessible on YouTube, many institutional firewalls prohibit access to YouTube and other web pages. As podcasts are available on nearly any Wi-Fi or cellular device and can be streamed from a variety of freely available platforms, content that is distributed in podcast form will be more accessible within healthcare institutions.

\section{Limitations}

The single greatest limitation to this study is its small sample size and small number of overall neurology-themed podcasts. This limitation is expected to be rectified by the more comprehensive analysis of the ADVANCE dataset which is underway. Although the survey response rate among neurology-themed programs was high (67\%), there are few neurology podcasts with generally infrequent episode publications and many episodes with small download counts by academic standards [18]. The small sample size raises the possibility of a type II error and precludes other exploratory analyses. However, many of these comparisons are being reported separately using the comprehensive ADVANCE dataset, which includes non-neurology-themed podcasts as well. The limited available data regarding podcast utilization, accessibility, duration, and user demographics also preclude certain potentially useful analyses. User location (e.g., country), user background (e.g., practicing professional, medical student, patient), and individual frequency of usage are not reported by standard podcast platforms. It is possible-although unlikely-that there was a shift in the type of users who accessed program content during the COVID-19 pandemic (e.g., fewer patients and more healthcare professionals), but we are unable to confirm this.

\section{Conclusions}

Despite the shift in medical education to the virtual classroom during the COVID-19 pandemic, there was no significant increase in neurology-themed podcast utilization early in the course of the pandemic. The COVID-19 pandemic may not have been a significant contributor to neuromedical podcast utilization, but we found that some podcast features (such as author interviews) may be more attractive among listeners, while other features (such as opportunities for CME credit) may not contribute significantly to podcast popularity. Ensuring widespread availability of podcast content using free platforms (such as iTunes and YouTube) can dramatically increase the utilization of these educational programs. We are actively exploring which program features and styles are most popular among medical listeners in a comprehensive analysis among neurology—and nonneurology-themed podcasts and blogs.

Author contribution Dr. Siegler conceived the study, collected the data, performed the statistical analyses, and wrote the manuscript. Dr. Boreski helped design the study methods, collected the data, interpreted the results, and provided critical feedback to the final manuscript. Dr. Strowd, Mr. Rook, Dr. Goss, Dr. Al-Mufti, Ms. Rossow, Dr. Miller, Ms. Chamberlain, Dr. London, Ms. Hurley, Dr. Geocadin, Dr. Richie, Dr. Isaacson, and Dr. Rybinnik collected the data, interpreted the results, and provided critical feedback to the final manuscript. Dr. Chan helped design the study methods, collected the data, interpreted the results, and provided critical feedback to the final manuscript. All the authors read and approved the final version of the manuscript.

Availability of data Data will be made available to any qualified investigator following ethical approval.

Code availability Statistical code (STATA 15.0.do file) will be made available to any qualified investigator.

\section{Declarations}

Ethics approval This study did not meet criteria for human subjects research; therefore, approval by the Institutional Review Board of the data coordinating center (Cooper University Hospital) was not required.

Consent for publication Not applicable.

Conflict of interest Dr. Siegler reports receiving fees from the private sector for the production of podcast materials and content (BrainWaves: A Neurology Podcast, Neurocritical Care Society Podcast), and has received honoraria for speaking about online educational resources. Dr. Strowd receives an editorial stipend as Deputy Section Editor of the Resident and Fellow Section of Neurology®. Dr. Chan has received a grant from the PSI Foundation, Inc., for her work on knowledge translation using social media. The remaining authors report no competing financial interests exist with this manuscript.

\section{References}

1. Hau H-M, Weitz J, Bork U (2020) Impact of the COVID-19 pandemic on student and resident teaching and training in surgical oncology. J Clin Med Res 9(11). https://doi.org/10.3390/jcm91 13431

2. Theoret C, Ming X (2020) Our education, our concerns: medical student education impact due to COVID-19. Med Educ 54(7):591-592

3. Ahmed H, Allaf M, Elghazaly H (2020) COVID-19 and medical education. Lancet Infect Dis 20(7):777-778

4. Sigdel S, Ozaki A, Dhakal R, Pradhan B, Tanimoto T (2020) Medical education in Nepal: impact and challenges of the COVID-19 pandemic. Acad Med. Published Online Ahead of Print. https:// doi.org/10.1097/ACM.0000000000003888 
5. Khamees D, Brown CA, Arribas M, Murphey AC, Haas MRC, House JB (2020) In crisis: medical students in the COVID-19 pandemic. AEM Educ Train 4(3):284-290

6. Rana T, Hackett C, Quezada T, Chaturvedi A, Bakalov V, Leonardo J et al (2020) Medicine and surgery residents' perspectives on the impact of COVID-19 on graduate medical education. Med Educ Online 25(1):1818439

7. Siegler JE, Heslin ME, Thau L, Smith A (2020) Falling stroke rates during COVID-19 pandemic at a comprehensive stroke center: Cover title: Falling stroke rates during COVID-19. J Stroke Cerebrovasc Dis. Available from: https://www.sciencedirect.com/ science/article/pii/S105230572030361X?casa_token=yXSRn HGIRDsAAAAA:mB131Mpc1HjgqUiSzc4APwrhkY21fiZjCKE zuNVcWA3s76EPwUjET1RoNRki8hZFBMhxPKhCHuM. Accessed 17 Feb 2021

8. Zhao J, Wang Y, Fisher M, Liu R (2020) Slower recovery of outpatient clinics than inpatient services for stroke and other neurological diseases after COVID-19 pandemic. CNS Neurosci Ther 26(12):1322-1326

9. Edigin E, Eseaton PO, Shaka H, Ojemolon PE, Asemota IR, Akuna E (2020) Impact of COVID-19 pandemic on medical postgraduate training in the United States. Med Educ Online 25(1): 1774318

10. Wilcha R-J (2020) Effectiveness of virtual medical teaching during the COVID-19 crisis: systematic review. JMIR Med Educ. 6(2):e20963

11. Ferrel MN, Ryan JJ (2020) The impact of COVID-19 on medical education. Cureus. 12(3):e7492

12. Thoma B, Goerzen S, Horeczko T, Roland D, Tagg A, Chan TM et al (2020) An international, interprofessional investigation of the self-reported podcast listening habits of emergency clinicians: a METRIQ Study. CJEM 22(1):112-117
13. Thoma B, Turnquist A, Zaver F, Hall AK, Chan TM (2019) Communication, learning and assessment: exploring the dimensions of the digital learning environment. Med Teach 41(4):385-390

14. AlGaeed M, Grewal M, Richardson PK, Leon Guerrero CR (2020) COVID-19: neurology residents' perspective. J Clin Neurosci 78:452-453

15. Chan TM, Stehman C, Gottlieb M, Thoma B (2020) A short history of free open access medical education. The past, present, and future. ATS Scholar 1(2):87-100

16. Siegler JE (2017) Media and book reviews. Neurology 88(15):e150-e152

17. Cadogan M, Thoma B, Chan TM, Lin M (2014) Free Open Access Meducation (FOAM): the rise of emergency medicine and critical care blogs and podcasts (2002-2013). Emerg Med J 31(e1):e76-e77

18. Cabrera D, Vartabedian BS, Spinner RJ, Jordan BL, Aase LA, Timimi FK (2017) More than likes and tweets: creating social media portfolios for academic promotion and tenure. J Grad Med Educ 9(4):421-425

19. Holm S (1979) A simple sequentially rejective multiple test procedure. Scand Stat Theory Appl 6(2):65-70

20 Paterson QS, Thoma B, Kenneth Milne W, Lin M, Chan TM (2015) A systematic review and qualitative analysis to determine quality indicators forhealth professions education blogs and podcasts. J Grad Med Educ 7:549-54. https://doi.org/10.4300/ jgme-d-14-00728.1

Publisher's note Springer Nature remains neutral with regard to jurisdictional claims in published maps and institutional affiliations. 